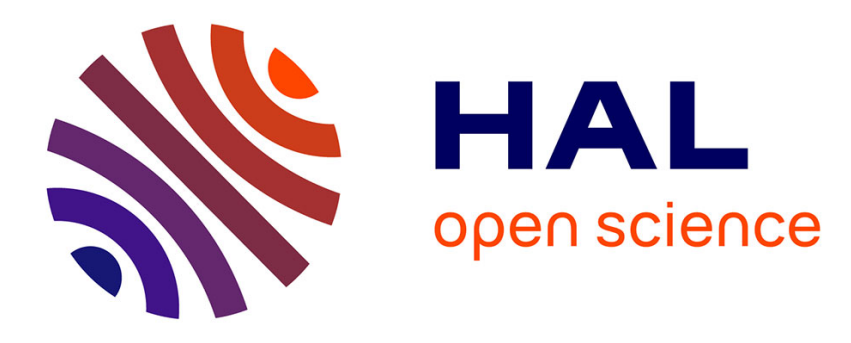

\title{
Batch sizing with controllable production rates
}

Christoph H. Glock

\section{To cite this version:}

Christoph H. Glock. Batch sizing with controllable production rates. International Journal of Production Research, 2009, 48 (20), pp.5921-5938. 10.1080/00207540903170906 . hal-00530443

\section{HAL Id: hal-00530443 \\ https://hal.science/hal-00530443}

Submitted on 29 Oct 2010

HAL is a multi-disciplinary open access archive for the deposit and dissemination of scientific research documents, whether they are published or not. The documents may come from teaching and research institutions in France or abroad, or from public or private research centers.
L'archive ouverte pluridisciplinaire HAL, est destinée au dépôt et à la diffusion de documents scientifiques de niveau recherche, publiés ou non, émanant des établissements d'enseignement et de recherche français ou étrangers, des laboratoires publics ou privés. 


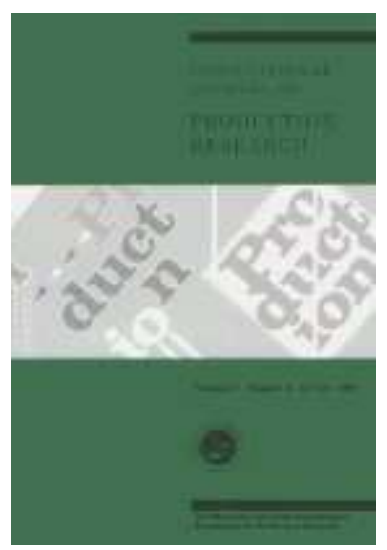

\section{Batch sizing with controllable production rates}

\begin{tabular}{|c|c|}
\hline Journal: & International Journal of Production Research \\
\hline Manuscript ID: & TPRS-2009-IJPR-0249.R1 \\
\hline Manuscript Type: & Original Manuscript \\
\hline $\begin{array}{r}\text { Date Submitted by the } \\
\text { Author: }\end{array}$ & 17-Jun-2009 \\
\hline Complete List of Authors: & $\begin{array}{l}\text { Glock, Christoph; University of Wuerzburg, Chair of Business } \\
\text { Management and Industrial Management }\end{array}$ \\
\hline Keywords: & INVENTORY CONTROL, INVENTORY MANAGEMENT, BATCH SIZING \\
\hline Keywords (user): & variable production rate \\
\hline
\end{tabular}

\section{s ScholarONE \\ Manuscript Central}




\title{
Batch sizing with controllable production rates
}

\author{
Christoph H. Glock \\ Chair of Business Management and Industrial Management \\ University of Wuerzburg \\ Sanderring 2 \\ 97070 Wuerzburg \\ Germany \\ Tel.: ++49931312408 \\ Fax: ++49 931312405 \\ E-Mail: christoph.glock@uni-wuerzburg.de
}

\begin{abstract}
In this paper, we focus on a production system where a single product is manufactured on a single facility and delivered to the subsequent stage in batch shipments. In contrast to earlier works, we assume that the inventory on the producing stage is depleted at discrete time intervals, and analyse the effect of a variable production rate on the inventory build-up and the total costs of the system. We develop formal models for the case of equal- and unequalsized batch shipments and propose solution procedures for the models. In a numerical study, we illustrated that deviating from the "design production rate" of a production system may reduce inventory carrying costs and thus lead to lower total costs.
\end{abstract}

Keywords: inventory control; production; variable production rate; batch sizing 


\section{Introduction}

The SCM-related literature contains a broad variety of models that deal with the control of inventories in different application areas. This is an important field of research, since it has been shown that the total inventory in a supply chain may exceed 100 days of supply (see Lee et al. 1997), wherefore the reduction of excess inventories may lead to many benefits. Lotsizing models, in this context, aim on balancing lot-proportional costs (i.e. costs that depend on the lot size, such as inventory carrying costs) and lot-fixed costs (i.e. fixed costs incurred with each lot produced, such as setup costs) by determining a production quantity for the system under study.

One major drawback we identified when studying the literature on lot-sizing is that the production rate of a facility is often treated as a constant and not as a decision variable. In contrast, the mean output per unit of time of a production system can be influenced in many practical situations, either by directly varying the rate of performance of the production equipment, or by inserting idle time between task elements and by thus varying the time which is effectively spent on manufacturing (see for example Buzacott and Ozkarahan, 1983; Schweitzer and Seidmann, 1991). Since the production rate can, for example, influence inventory build-up or the life-span of the tools used in the manufacturing process, controlling the production rate may help to reduce the total costs of the system under study.

In recent years, several authors have studied the effect of a variable production rate on the performance of inventory systems. Thereby, some authors permit the production rate to be changed during the production process, while other authors assume that the production rate has to be fixed before a production run starts. The first case, which is commonly referred to as the "flexible case", may occur if setup costs are only incurred when switching to a different item, but not when adjusting the speed of the production equipment, while the latter, which is termed the "rigid case", may occur if changing the setup of a machine during a production run is technically impossible or associated with prohibitively high costs.

One of the first models dealing with the rigid case is due to Buzacott and Ozkarahan (1983), who consider the problem of scheduling two products on a single or two machines. The authors assume that the production rate can be varied by inserting idle time over the production run, and show that it is optimal to choose the production rates for both models in such a way that the machines are always fully utilised, i.e. that no inserted idle time occurs between runs of the products.

A variation of the model is introduced by Silver (1990), who analyzes the production of a family of items on a single facility. The author assumes that the products are produced once in 
a common production cycle, and permits the production rate to be varied between prescribed limits. In contrast to Buzacott and Ozkarahan (1983), operating costs which occur per unit operating time are considered in the model formulation. Thus, since running the production equipment is no longer free of cost, it is not generally optimal to avoid idle time on the machine.

The effect of a variable production rate on the production of items with a limited shelf life is first analyzed by Sarker and Babu (1993). The authors show that in case certain products can be stored in the inventory only for a limited time span, it may be beneficial either to reduce the production cycle or the production rate to shorten the storage time of the products and to avoid spoilage. Extensions to the model can be found in Silver (1995), Goyal (1994), Viswanathan (1995), and Viswanathan and Goyal (1997), who permit a simultaneous reduction of both model parameters or a different scheduling strategy, among others.

Larsen (1997) and Sharma (2008) study both a variable demand and a variable production rate in an EPQ-model. Both authors assume that the demand rate may fluctuate, and analyze how the production rate should be adjusted to compensate these fluctuations. It is shown that the appropriate response to an increased demand rate is a reduction of the production rate, which leads to reduced inventory build-up and consequently lower inventory carrying costs.

The effect of a variable production rate on product quality is analyzed by Khouja and Mehrez (1994) and Khouja (1999). Khouja and Mehrez (1994) assume that product quality deteriorates with an increased production rate and integrate different production cost and quality functions into an EPQ-model. The authors show that in case an increased production rate leads to a significant decrease in quality, the optimal production rate may be smaller than the rate that minimises unit production cost. Similarly, Khouja (1999) assumes that the production process may shift out of control with a probability depending on the production rate. The author extends the model proposed by Silver (1990) and shows that incorporating quality leads to a reduction in the optimal lot sizes and cycle time.

The flexible case is first analyzed by Moon et al. (1991), who extend the model due to Silver (1990) to allow production rates to be varied during the production run. The authors show that it is optimal first to reduce the production rate of a particular item to meet the demand rate in order to avoid inventory build-up, and then to produce the item at its maximum rate to build enough inventory to satisfy demand until the item is produced again. Since the authors do not consider production costs, there is no idle time in the optimal schedule. An extension to the model is provided by Gallego (1993), who applies a power-of-two-policy, instead of a common cycle-policy, and shows that in minimising a lower bound on the average cost it is 
optimal to reduce the production rate of at most one item. A further extension is introduced by Elhafsi and Bai (1997), who allow backordering in their model and show that varying the production rate might again lead to a substantial reduction in average cost.

An EPQ-model which considers the flexible case is finally provided by Larsen (2005), who assumes that the production rates and their corresponding run times are decision variables. The author shows that the production rates should be chosen in the interval between the demand rate and the production rate which minimises unit production costs, and that they should be used in an increasing order.

In this paper, we focus on the case where a single product is manufactured on a single facility and delivered to the subsequent stage in batch shipments. In contrast to earlier works, we assume that the inventory on the producing stage is depleted at discrete time intervals, and analyze the effect of a variable production rate on the inventory build-up and the total costs of the system. Thereby, we illustrated that deviating from the "design production rate" of a production system may reduce inventory in the system and thus lead to lower total costs. In conformity with the literature, we analyze both the "rigid case" and the "flexible case" in the following and study their impact on the system for the cases of equal- and unequal-sized batch shipments.

Our research is especially important for companies facing high in-process inventories or bottleneck-problems. As will be shown, varying the production rate of a machine may significantly influence waiting time-related inventory and may thus help to control queues in front of subsequent production stages. This is consistent with the OPT-Philosophy (see for example Goldratt 1988), which aims on maximizing the throughput of the production system as a whole, instead of the throughput of a single machine, and the JIT-philosophy, which aims on reducing in-process inventories.

The remainder of the paper is organised as follows: in the next section, the article describes the problem we focus and outlines the assumptions and definitions which will used in the remaining sections of the paper. Accordingly, we develop formal models for the rigid and the flexible case and the case of equal- and unequal-sized batch shipments and propose solution procedures for the models. Section 4 contains a numerical study and section 5 concludes the article.

\section{Problem description}

In the following, we study a system where a single product is manufactured on a single facility and assume that partial shipments to the subsequent stage can be made before the 


\section{Assumptions:}

1. All parameters are deterministic and constant over time.

2. The production rate can be varied between given limits $p_{\min }$ and $p_{\max }$.

3. We exclude synchronous production, where the facility is constantly producing and where there is no setup, from the analysis and assume that $p_{\min }>d$.

4. The unit production costs are a function of the production rate.

5. The unit production cost function is convex in $p$ and $p_{0}$ is the "design production rate" that minimises unit production costs.

6. Batches are only sent to the consuming stage when the inventory level at this stage reaches zero.

7. We explicitly focus on a time weighted cost function to enhance the practical applicability of our model. As Higgins et al. (1996) note, production plans are often made with planning horizons rolling through time as time progresses. Thus, companies who wish to implement our model as a heuristic planning tool may specify a planning period and repeat the planning process after the planning time has elapsed. Note that this assumption is not uncommon in the literature, cf. for example Bogaschewsky et al. (2001). However, normalizing the planning period to 1 time unit results in the common money-per-unit-time cost function.

8. Shortages are not allowed. 


\section{Notations:}

$c_{p}(p)=$ unit production cost function

$c_{S}=$ setup costs per setup

$c_{T}=$ transportation costs per shipment

$d=$ demand rate in units per unit time

$D=$ total demand in the planning period

$h=$ inventory carrying charges per unit per unit of time

$q_{j}=$ size of the $j$ th batch shipment

$\lambda=$ the proportional increase in successive shipments within a batch production run

$m=$ number of batch shipments in a production cycle

$p=$ production rate in units per unit time

$p_{0}=$ design production rate

$Q=$ production lot size

\section{Definitions:}

$I^{\text {reg }}=$ "regular" inventory

$I^{\text {wait }}=$ inventory due to waiting times

$I C=$ inventory carrying costs

$P C=$ production costs

$T C=$ total costs in the planning period

$T W I=$ time weighted inventory

\section{Model formulation and solution}

\section{The rigid case}

\section{a) Equal-sized batch shipments}

The first scenario we analyze is the case where equal-sized batch shipments are transported to the subsequent stage and where the production rate may be varied only before the start of a production run. The corresponding inventory time plots are illustrated in figure 1 (cf. the bold lines. Note that the dashed lines represent the case of a reduced production rate). It is shown that the producing stage manufactures a lot of size $Q$ in $m t_{p}$ time units, and that the first batch is shipped to the subsequent stage directly after its completion. Due to $p>d$, the second batch is finished before the first batch is completely used up, wherefore it has to be kept in stock for $\mathrm{t}_{\mathrm{w}, 1}=t_{v}-t_{p}$ time units. 
Figure 1

The time weighted inventory in this case consists of a "regular" inventory, which corresponds to the triangles shown in figure 1, and inventory due to waiting times. The "regular" inventory can be expressed as follows:

(1) $I^{r e g}=\frac{Q^{2}}{2 m}\left(\frac{1}{p}+\frac{1}{d}\right)$

Inventory due to waiting times can be calculated as:

$$
I^{\text {wait }}=\frac{Q^{2}}{m^{2}} \sum_{i=2}^{m} i\left(\frac{1}{d}-\frac{1}{p}\right)=\frac{Q^{2}}{2 m}(m-1)\left(\frac{1}{d}-\frac{1}{p}\right)
$$

The time weighted inventory is given as the sum of (1) and (2). The inventory carrying costs in the planning period thus equal:

(3) IC $=\frac{D Q}{2 m}\left(\frac{1}{p}(2-m)+\frac{m}{d}\right) h$

Apart from the inventory carrying costs, the system encounters production costs which vary with a varying production rate. In the following, we assume that the unit production cost function is of the form specified by Eiamkanchanalai and Banerjee (1999), wherefore we can conclude that:

(4) $\quad P C=D c_{p}(p)=D\left(a_{0} p^{2}-a_{1} p+a_{2}\right)$

The total costs consist of the sum of (3) and (4) and the setup and transportation costs in the planning period, which amount to $\left(c_{S}+m c_{T}\right) D / Q$. Thus, it follows that:

$$
T C=\frac{D Q}{2 m}\left(\frac{m}{d}+\frac{2-m}{p}\right) h+\left(c_{S}+m c_{T}\right) \frac{D}{Q}+D c_{p}(p)
$$

As might be easily shown, (5) is convex in $Q$ for given values for $m$ and $p$. Thus, the optimal solution for $Q$ is given as:

$$
Q^{*}=\sqrt{\frac{2 m\left(c_{S}+m c_{T}\right)}{\left(\frac{m}{d}+\frac{2-m}{p}\right) h}}
$$

Substituting (6) in (5), the total cost function reduces to:

$$
T C=D \sqrt{\frac{2}{m}\left(\frac{m}{d}+\frac{2-m}{p}\right) h\left(c_{S}+m c_{T}\right)}+D c_{p}(p)
$$


As could be shown by Szendrovits and Drezner (1980), (7) is quasi-convex in $m$. Thus, for a given value for $p$, an optimal solution for $m$ may be calculated with the help of the following optimality condition:

(8) $T C\left(m^{*}-1\right) \geq T C\left(m^{*}\right) \leq T C\left(m^{*}+1\right)$

However, since (7) is not necessarily convex in $p$, we state the following theorems to derive a solution:

Theorem 1: For $m=1$, producing with $p<p_{0}$ cannot be optimal.

Proof: see appendix 1.

Theorem 2: For $m=2$, it is optimal to produce with $p=p_{0}$.

Proof: see appendix 2.

Theorem 3: For $\mathrm{m}>2$, producing with $p>p_{0}$ cannot be optimal.

Proof: see appendix 3.

To calculate a good, but not necessarily optimal, value for $p$, we can apply a one-dimensional line search algorithm. The following procedure can be used to find a solution for $m^{*}, p^{*}$, and $Q^{*}:$

Step 1: Set $m=1$ and search $p \in\left[p_{0} ; p_{\max }\right]$ that minimises $T C$. Set $T C^{*}=T C, m^{*}=m$, and $p^{*}$ $=p$.

Step 2: Set $m=2$ and $p=p_{0}$. If $T C>T C^{*}$, Goto Step 4 .

Set $T C^{*}=T C, m^{*}=m, p^{*}=p$, and $m=m+1$

Step 3: Search $p \in\left[p_{\min } ; p_{0}\right]$ that minimises $T C$. If $T C>T C^{*}$, Goto Step 4.

Set $T C^{*}=T C, m^{*}=m, p^{*}=p$, and $m=m+1$. Goto Step 3 .

Step 4: Find $Q^{*}$ from (6).

b) Unequal-sized batch shipments

In the case where unequal-sized batches are shipped to the subsequent stage, the size of the $j$ th batch can be calculated as follows (see Goyal, 1977):

(9) $\quad q_{j}=q_{1} \lambda^{j-1}=q_{1}(p / d)^{j-1} \quad$ with $Q=\sum_{i=1}^{m} q_{i}=q_{1} \sum_{i=1}^{m} \lambda^{i-1}$ 
The corresponding inventory time plots that result if $t_{p, j}=t_{v, j-1}$ are illustrated in figure 2 (cf. the bold lines. The dashed lines again illustrate the case of a reduced production rate). As can be seen, there is no inventory due to waiting times if subsequent batches increase by $\lambda=p / d$.

\section{Figure 2}

The time weighted inventory can be calculated as follows:

$$
\begin{aligned}
T W I & =\frac{q_{1}}{2}\left(\frac{q_{1}}{p}+\frac{q_{1}}{d}\right)+\frac{q_{2}}{2}\left(\frac{q_{2}}{p}+\frac{q_{2}}{d}\right)+\ldots+\frac{q_{m}}{2}\left(\frac{q_{m}}{p}+\frac{q_{m}}{d}\right)= \\
& =\frac{q_{1}^{2}}{2}\left(\frac{1}{p}+\frac{1}{d}\right) \sum_{i=1}^{m}\left(\lambda^{i-1}\right)^{2}=\frac{q_{1}^{2}}{2}\left(\frac{1}{p}+\frac{1}{d}\right) \frac{\lambda^{2 m}-1}{\lambda^{2}-1}
\end{aligned}
$$

The total costs can now be derived if the time weighted inventory is multiplied with the number of lots in the planning period $(D / Q)$ and the unit inventory carrying charges per unit of time $h$, and if the setup costs per order and the transportation costs per batch are considered in addition:

$$
T C=\frac{D q_{1}}{2}\left(\frac{1}{p}+\frac{1}{d}\right) \frac{\lambda^{m}+1}{\lambda+1} h+\left(c_{S}+m c_{T}\right) \frac{D(\lambda-1)}{q_{1}\left(\lambda^{m}-1\right)}+D c_{p}(p)
$$

Note that (11) equals (5) for $\lambda=1$ and $q_{1}=Q / m$. Since (11) is convex in $q_{1}$ for given values for $m$ and $p$, a solution for the optimal first batch size can be calculated with the help of differential calculus. It follows:

$$
q_{1}^{*}=\sqrt{\frac{2(\lambda-1)(\lambda+1)\left(c_{S}+m c_{T}\right)}{\left(\lambda^{m}-1\right)\left(\lambda^{m}+1\right)\left(\frac{1}{p}+\frac{1}{d}\right) h}}
$$

Substituting (12) in (11), the total cost function reduces to:

$$
T C=D \sqrt{2 h\left(c_{S}+m c_{T}\right)\left(\frac{1}{d}-\frac{1}{p}\right) \frac{\left(\frac{p}{d}\right)^{m}+1}{\left(\frac{p}{d}\right)^{m}-1}}+D c_{p}(p)
$$

Since (13) has a unique minimum in $m$ for given values of $p$ (see Goyal, 1977), an optimal solution may be derived with the help of the following optimality condition:

$$
T C\left(m^{*}-1\right) \geq T C\left(m^{*}\right) \leq T C\left(m^{*}+1\right)
$$

However, (13) is not necessarily convex in $p$. To derive a solution for the production rate, we state the following theorem and proposition: 
Theorem 4: For $m=1$, producing with $p<p_{0}$ cannot be optimal.

Proof: follows directly from theorem 1.

Proposition 1: Assume that $\hat{p}$ is the production rate that minimises inventory carrying cost. If $\left.\hat{p}\right|_{m=2}>p_{0}$, the optimal solution for $p$ is contained in the interval $\left[p_{\min } ; d(1+\sqrt{2})\right]$, and otherwise in the interval $\left[p_{\min } ; p_{0}\right]$

Explanation: see appendix 4.

To calculate a good, but not necessarily optimal, value for $p$, we can again apply a onedimensional line search algorithm over the intervals identified above. The following procedure can be used to find a solution for $m^{*}, p^{*}$, and $q_{1} *$ :

Step 1: Set $m=1$ and search $p \in\left[p_{0} ; p_{\max }\right]$ that minimises $T C$. Set $T C^{*}=T C, m^{*}=m$, and $p^{*}$ $=p$. Set $m=m+1$.

Step 2: Search $p \in\left[p_{\min } ; \operatorname{Max}\left[p_{0}, d(1+\sqrt{2})\right]\right]$ that minimises $T C$. If $T C>T C^{*}$, Goto Step 3 . Set $T C^{*}=T C, m^{*}=m, p^{*}=p$, and $m=m+1$. Goto Step 2 .

Step 3: Find $q_{1}^{*}$ from (12).

\section{The flexible case}

\section{a) Equal-sized batch shipments}

So far, we assumed that the production rate has to be fixed before a production run starts. In the following, we relax this assumption and suppose that each batch may be produced with a separate production rate. In compliance with earlier work, we assume that the costs of changing the production rate are negligible.

If each batch is manufactured with a separate production rate, the "regular" inventory given in (1) has to be reformulated as follows:

$$
I^{r e g}=\frac{Q^{2}}{2 m^{2}}\left(\frac{m}{d}+\sum_{i=1}^{m} \frac{1}{p_{i}}\right)
$$

Similarly, inventory due to waiting times can be calculated as:

$$
I^{\text {wait }}=\frac{Q}{m}\left(\sum_{i=2}^{m}(i-1) \frac{Q}{d}-\sum_{j=2}^{i} \frac{Q}{p_{j}}\right)=\frac{Q^{2}}{2 m^{2}}\left(m(m-1) \frac{1}{d}-2 \sum_{i=2}^{m} \sum_{j=2}^{i} \frac{1}{p_{j}}\right)
$$

The time weighted inventory is again given as the sum of (15) and (16). The inventory carrying costs in the planning period thus equal: 


$$
I C=\frac{Q^{2}}{2 m^{2}}\left(\frac{m^{2}}{d}+\sum_{i=1}^{m} \frac{1}{p_{i}}-2 \sum_{i=2}^{m} \sum_{j=2}^{i} \frac{1}{p_{j}}\right) h=\frac{Q^{2}}{2 m^{2}}\left(\frac{m^{2}}{d}+\frac{1}{p_{1}}-\sum_{i=2}^{m}\left(\sum_{j=2}^{i-1} \frac{1}{p_{j}}+\sum_{j=2}^{i} \frac{1}{p_{j}}\right)\right) h
$$

Considering production costs and setup and transportation costs leads to the total cost function:

$$
T C=\frac{D Q}{2 m^{2}}\left(\frac{m^{2}}{d}+\frac{1}{p_{1}}-\sum_{i=2}^{m}\left(\sum_{j=2}^{i-1} \frac{1}{p_{j}}+\sum_{j=2}^{i} \frac{1}{p_{j}}\right)\right) h+\left(c_{S}+m c_{T}\right) \frac{D}{Q}+\frac{D}{m} \sum_{i=1}^{m} c_{p}\left(p_{i}\right)
$$

Since (18) is convex in $Q$ for given values for $m$ and a given $p$-vector, the optimal solution for $Q$ is given as follows:

$$
Q^{*}=\sqrt{\frac{2 m^{2}\left(c_{S}+m c_{T}\right)}{\left(\frac{m^{2}}{d}+\frac{1}{p_{1}}-\sum_{i=2}^{m}\left(\sum_{j=2}^{i-1} \frac{1}{p_{j}}+\sum_{j=2}^{i} \frac{1}{p_{j}}\right)\right) h}}
$$

Substituting (19) in (18) leads to:

$$
T C=\frac{D}{m}\left(\sqrt{2\left(c_{S}+m c_{T}\right)\left(\frac{m^{2}}{d}+\frac{1}{p_{1}}-\sum_{i=2}^{m}\left(\sum_{j=2}^{i-1} \frac{1}{p_{j}}+\sum_{j=2}^{i} \frac{1}{p_{j}}\right)\right) h}+\sum_{i=1}^{m} c_{p}\left(p_{i}\right)\right)
$$

Since (20) is not necessarily convex in $p_{i}$ and $m$, we state the following theorems to derive a good solution:

Theorem 5: Producing the first batch with $p_{1}<p_{0}$ cannot be optimal. Producing the batches 2 to $m$ with $p_{i}>p_{0}$ cannot be optimal.

Proof: see appendix 5.

Theorem 6: For $j>k, p_{j}<p_{k}$ cannot be optimal.

Proof: see appendix 5.

To calculate a good, but not necessarily optimal, value for $p_{i}$, we can again apply a line search algorithm over the intervals identified above. Further, we calculate a solution for $m$ with the help of the following condition:

$$
T C\left(m^{*}-1\right) \geq T C\left(m^{*}\right) \leq T C\left(m^{*}+1\right)
$$

The following procedure can be applied to find a solution for $m^{*}, Q^{*}$, and the $p$-vector:

Step 1: Set $m=1$ and search $p_{1} \in\left[p_{0} ; p_{\max }\right]$ that minimises $T C$. Set $T C^{*}=T C, m^{*}=m$, and $p_{1}^{*}=p_{1}$. Set $m=m+1$. 
Step 2: Search $p_{j} \in\left[p_{\min } ; \operatorname{Max}\left[p_{0}, d(1+\sqrt{2})\right]\right] \forall j \in[2, \ldots, m]$ that minimises $T C$. If $T C>T C^{*}$, Goto Step 3.

Set $T C^{*}=T C, m^{*}=m, p_{j}^{*}=p_{j} \forall j \in[2, \ldots, m]$, and $m=m+1$. Goto Step 2.

Step 3: Find $q_{1}$ from (12).

\section{b) Unequal-sized batch shipments}

In the case where unequal-sized batches are transported to the subsequent stage, the size of the $j$ th batch depends on the size of the first shipment and the production rate of batches 2 to $j$ (cf. figure 3). Thus, it follows that

$$
q_{j}=q_{1} \prod_{i=2}^{j} \frac{p_{i}}{d} \quad \text { and } \quad \sum_{j=1}^{m} q_{j}=q_{1} \sum_{j=1}^{m} \prod_{i=2}^{j} \frac{p_{i}}{d}
$$

\section{Figure 3}

The time weighted inventory can be calculated as follows:

$$
\begin{aligned}
T W I & =\frac{q_{1}}{2}\left(\frac{q_{1}}{p_{1}}+\frac{q_{1}}{d}\right)+\frac{q_{2}}{2}\left(\frac{q_{2}}{p_{2}}+\frac{q_{2}}{d}\right)+\ldots+\frac{q_{m}}{2}\left(\frac{q_{m}}{p_{m}}+\frac{q_{m}}{d}\right)= \\
& =\frac{q_{1}^{2}}{2}\left(\frac{1}{p_{1}}+\frac{1}{d}\right)+\frac{q_{1}^{2}}{2}\left(\frac{p_{2}}{d}\right)^{2}\left(\frac{1}{p_{2}}+\frac{1}{d}\right)+\ldots+\frac{q_{1}^{2}}{2}\left(\prod_{i=2}^{m} \frac{p_{i}}{d}\right)^{2}\left(\frac{1}{p_{m}}+\frac{1}{d}\right)= \\
& =\frac{q_{1}^{2}}{2} \sum_{i=1}^{m}\left(\prod_{j=2}^{i} \frac{p_{j}}{d}\right)^{2}\left(\frac{1}{p_{i}}+\frac{1}{d}\right)
\end{aligned}
$$

The total costs are again given if the time weighted inventory is multiplied with the number of lots in the planning period $\left(D / \sum q_{i}\right)$ and the unit inventory carrying charges per unit of time $h$, and if the setup costs per order and the transportation costs per batch are considered in addition:

$$
T C=\frac{D}{\sum_{j=1}^{m} \prod_{i=2}^{j} \frac{p_{i}}{d}}\left(\frac{q_{1} h}{2} \sum_{i=1}^{m}\left(\prod_{j=2}^{i} \frac{p_{j}}{d}\right)^{2}\left(\frac{1}{p_{i}}+\frac{1}{d}\right)+c_{S}+m c_{T}+\sum_{i=1}^{m} c_{p}\left(p_{i}\right) \prod_{j=2}^{i} \frac{p_{j}}{d}\right)
$$

Since (24) is convex in $q_{1}$ for given values for $m$ and $p_{i}$, the optimal solution for $q_{1}$ is given as:

$$
q_{1}^{*}=\sqrt{\frac{2\left(c_{S}+m c_{T}\right)}{\sum_{i=1}^{m}\left(\prod_{j=2}^{i} \frac{p_{j}}{d}\right)^{2}\left(\frac{1}{p_{i}}+\frac{1}{d}\right) h}}
$$

Substituting (25) in (24) leads to: 


$$
T C=\frac{D}{\sum_{j=1}^{m} \prod_{i=2}^{j} \frac{p_{i}}{d}}\left(\sqrt{2\left(c_{S}+m c_{T}\right) h \sum_{i=1}^{m}\left(\prod_{j=2}^{i} \frac{p_{j}}{d}\right)^{2}\left(\frac{1}{p_{i}}+\frac{1}{d}\right)}+\sum_{i=1}^{m} c_{p}\left(p_{i}\right) \prod_{j=2}^{i} \frac{p_{j}}{d}\right)
$$

Since (26) is not necessarily convex in $p_{i}$ and $m$, we state the following theorems to derive a good solution:

Theorem 7: Producing the first batch with $p_{1}<p_{0}$ cannot be optimal. Producing batches 2 to $m$ with $p_{i}>p_{0}$ cannot be optimal.

Proof: see appendix 6.

To calculate a good, but not necessarily optimal, value for $p_{i}$, we can again apply a line search algorithm over the intervals identified above. Further, we calculate a solution for $m$ with the help of the following condition:

(21) $T C\left(m^{*}-1\right) \geq T C\left(m^{*}\right) \leq T C\left(m^{*}+1\right)$

The following procedure can be applied to find a solution for $m^{*}, q_{1}^{*}$, and the $p$-vector:

Step 1: Set $m=1$ and search $p_{1} \in\left[p_{0} ; p_{\max }\right]$ that minimises $T C$. Set $T C^{*}=T C, m^{*}=m$, and $p_{1}^{*}=p_{1}$. Set $m=m+1$.

Step 2: Search $p_{j} \in\left[p_{\min } ; p_{j-1, \max }\right] \forall j \in[2, \ldots, m]$ with $p_{2, \max }=p_{0}$ that minimises $T C$. If $T C>$ $T C^{*}$, Goto Step 3.

Set $T C^{*}=T C, m^{*}=m, p_{j}^{*}=p_{j} \forall j \in[2, \ldots, m]$, and $m=m+1$. Goto Step 2 .

Step 3: Find $q_{1}^{*}$ from (12).

\section{Numerical Examples}

To illustrate the effect of a variable production rate on a two-stage production system with discrete inventory depletions, we solved a set of test problems shown in table 1 . In addition to the data given below, we assumed that $D=1000$ and $c_{S}=250$. Each of these test problems were solved using the procedures described above. The results for the rigid case are given in tables 2 and 3 and the results for the flexible case in tables 4 and 5 .

Table 1

For problem one, reducing the production rate resulted in lower total costs if the rigid case was assumed. As can be seen, the reduction in $p$ is smaller in case unequal-sized batch 
shipments are transported to the subsequent stage. This is due to the fact that unequal-sized batch shipments reduce the total inventory in the system, wherefore a reduction in $p$ leads to a relatively smaller reduction in inventory carrying costs. Providing that each batch may be produced with a separate production rate, we found a major difference between the two shipment policies analyzed above: in case equal-sized batch shipments are transported to the subsequent stage, the batches follow the pattern identified above, i.e. $p_{2} * \leq p_{3}^{*} \leq \ldots \leq p_{m}{ }^{*} \leq$ $p_{1}{ }^{*}$. For the case of unequal-sized batch shipments, we found that the production rate of the first batch is increased with $p_{1}>p_{0}$, and that the production rate of subsequent batches is at first gradually reduced and then increased again. However, $p_{m}<p_{0}$ holds. This effect can be explained as follows: If the number of shipments that succeed a particular batch $i$ with $i \geq 2$ is large, a high production rate for batch $i$ leads to an increase of the respective and all subsequent batch sizes (cf. expression (22)). This effect is enhanced if the size of batch $i$ is large. Thus, a large difference between $m$ and $i$ and a large batch size $q_{i}$ favour a low production rate. If the difference $m-i$ is reduced, the production rate is increased again. Finally, it can be seen that the flexible case leads to lower total costs than the rigid case for all problems we tested, which supports the findings of prior studies.

Table 2

Problems two and three illustrate the effect of a variation of the inventory carrying charges per unit per unit of time on the selection of the production rate. An increase in $h$ leads to higher inventory carrying costs, which enhances the advantage that results from varying the production rate. In contrast, if $c_{T}$ is increased, the number of batch shipments and the proportion of inventory due to waiting times in the total inventory are decreased. This leads to a lower variation in $p_{i}$ in the rigid case and a higher production rate for the first batch in case different production rates are allowed for each batch shipment.

Table 3

The impact that results from varying the shape of the unit production cost function is illustrated in problems five and six. If the function is compressed, increasing or decreasing the production rate leads to a sharp increase in $P C$. Thus, the higher the slope of the unit production cost function, the lower the variation in $p_{i}$ for both the rigid and the flexible case. 
Table 4

Finally, problems seven and eight illustrate the effect of a variation in the interval of feasible production rates.

\section{Table 5}

\section{Conclusion}

In this paper, we considered a two-stage production system with equal- and unequal-sized batch shipments. We extended earlier work by treating the production rate as a decision variable and analyzed the resulting problem for both the cases where the production rate is fixed and variable during a production run. In a numerical study, we illustrated that deviating from the "design production rate" of a production system may reduce inventory carrying costs and thus lead to lower total costs. In order to increase the scope of our analysis, the model presented in this article could be extended to include costs of changing the production rate or a different unit production cost function (see for example Khouja (1995) or Moon and Christy (1998)).

\section{Appendices}

\section{Appendix 1}

For $m=1$, (7) reduces to:

$$
\text { (A-1) } \quad T C=D \sqrt{2\left(\frac{1}{d}+\frac{1}{p}\right) h\left(c_{S}+c_{T}\right)}+D c_{p}(p)
$$

As can be seen, the first addend in (A-1) decreases with an increasing production rate, while the second increases for both an increasing and a decreasing production rate. Consequently, it cannot be optimal to produce with a production rate smaller than $p_{0}$, since there is always a $p$ $\in\left[p_{0,} p_{\max }\right.$ that leads to lower total costs.

\section{Appendix 2}

For $m=2,(7)$ reduces to: 
$(\mathrm{A}-2) \quad T C=D \sqrt{\frac{2}{d} h\left(c_{S}+2 c_{T}\right)}+D c_{p}(p)$

As can be seen, the first addend in (A-2) is independent of $p$, while the second increases for both an increasing and a decreasing production rate. Consequently, (A-2) is minimised with $p$ $=p_{0}$.

To explain this effect, it is first necessary to analyze the variation of $I^{\text {reg }}$ and $I^{\text {wait }}$ for the case that $p$ is varied to $p$ '. It follows that:

$$
\begin{aligned}
& \Delta I^{\text {reg }}=I^{\text {reg }}(p)-I^{\text {reg }}\left(p^{\prime}\right)=\frac{Q^{2}}{2 m}\left(\frac{1}{p}-\frac{1}{p^{\prime}}\right) \\
& \Delta I^{\text {wait }}=I^{\text {wait }}(p)-I^{\text {wait }}\left(p^{\prime}\right)=\frac{Q^{2}}{2 m}(m-1)\left(\frac{1}{p^{\prime}}-\frac{1}{p}\right)
\end{aligned}
$$

The sum of (A-3) and (A-4) equals:

$$
\text { (A-5) } \Delta I=\Delta I^{\text {reg }}+\Delta I^{\text {wait }}=\frac{Q^{2}}{2}\left(\frac{2}{m}-1\right)\left(\frac{1}{p}-\frac{1}{p^{\prime}}\right)
$$

For $m=2$, (A-5) equals 0 . It follows that in case $m=2$, a variation in $p$ leads to a decrease (increase) in $I^{\text {reg }}$ that is exactly compensated by an increase (decrease) in $I^{\text {wait }}$ (it can be seen in figure 1 by comparing the bold and dashed lines that a decrease in $p$ leads to a higher regular inventory and a lower inventory due to waiting times). Thus, since $c_{p}(p)$ increases with an increasing or a decreasing production rate, it is optimal to produce with $p=p_{0}$.

\section{Appendix 3}

It is obvious that the expression $(2-m) / p$ in (7) is negative for $m>2$. Thus, the first addend in (7) can be reduced by lowering the production rate.

To explain this effect, we analyze the variation of $I^{\text {reg }}$ and $I^{\text {wait }}$ for the case that $p$ is varied to $p$ '. As can be seen in (A-3) and (A-4), an increase in $p$ leads to a decrease in $I^{\text {reg }}$ and an increase in $I^{\text {wait }}$. To assess the total effect, we refer to (A-5), which is positive for $m>2$ and $p$, $<p$. Thus, we conclude that in case $m>2$, a reduction in $p$ leads to an increase in $I^{\text {reg }}$, which is always compensated by a decrease in $I^{\text {wait }}$. Further, it follows that a decrease in $p$ is especially beneficial for high values for $m$, since the multiplier $(2 / m-1)$ enhances the effect of manufacturing with a reduced production rate for high values for $m$. However, the marginal decrease in inventory which results if $p$ is reduced to $p$ ' decreases with an increase in $m$.

\section{Appendix 4}

Minimising inventory carrying costs is equivalent to minimising 


\section{Figure 5}

As can be seen, $\alpha$ increases with an increasing production rate and approaches $1 / d$ if $p$ approaches infinity. In contrast, $\beta$ is negatively correlated with $p$ and approaches 1 if $p$ approaches infinity. If $m$ approaches infinity, $\beta$ approaches -1 for $p<d$ and 1 for $p>d$. Further, we conclude that a decrease in $\beta$, which occurs if $p$ is increased to $p$, is larger for $m$ than $m$ ' with $m<m^{\prime}$.

If $m=2,(\mathrm{~A}-6)$ is convex in $p$ and $\hat{p}$ minimises (A-6) with

$$
\text { (A-7) } \hat{p}=d(1+\sqrt{2})
$$

If $m>2$, it is difficult to derive a closed-form solution for $\hat{p}$. However, we identified (A-6) to be quasi-convex in $p$ with the form shown in figure 4 in numerical studies. Further, we conclude that $\hat{p}(m)>\hat{p}(m+1)$ and $\hat{p}(m \rightarrow \infty)=d$ due to the relations identified above.

\section{Figure 4}

Figure 5 illustrates the range of possible values for $p$ that contains the optimal solution. Thus, if $\left.\hat{p}\right|_{m=2}>p_{0}$, the value for $p$ that minimises (13) can be found in the interval $\left[p_{\min } ; d(1+\sqrt{2})\right]$, and otherwise in the interval $\left[p_{\min } ; p_{0}\right]$.

\section{Appendix 5}

Minimising inventory carrying costs in $p_{i}$ is equivalent to minimising

(A-8) $\frac{1}{p_{1}}-\sum_{i=2}^{m}\left(\sum_{j=2}^{i-1} \frac{1}{p_{j}}+\sum_{j=2}^{i} \frac{1}{p_{j}}\right)$

As can be easily seen, both minuend and subtrahend decrease with an increasing production rate. Thus, (A-8) can be minimised by increasing $p_{1}$ and decreasing $p_{2}, \ldots, p_{m}$. In addition, it can be seen that the sum function in the subtrahend leads to a higher weight for the production rate of earlier batch shipments, which is due to the fact that the production rate of batch $j$ with $j>1$ influences the waiting times of all subsequent batch shipments. Thus, since a variation in 
$p_{i}$ always leads to higher unit production costs, we conclude that $p_{1}^{*} \in\left[p_{0}, p_{\max }\right]$ and $p_{i}{ }^{*} \in$ $\left[p_{\min } ; p_{0}\right]$ for $i>1$. Further, due to the relations identified above, we conclude that $p_{2} * \leq p_{3} * \leq$ $\ldots \leq p_{m}^{*}$.

\section{Appendix 6}

As can be seen in figures 2 and 3, $p_{1}$ influences the inventory build-up of the first batch, but not the size of the shipments 2 to $m$. Thus, a decrease (increase) in $p_{1}$ leads to a slower (faster) inventory build-up and consequently higher (lower) inventory carrying costs. Since unit production costs are convex in $p_{i}$ with a unique minimum in $p_{0}$, it cannot be optimal to produce with a production rate $p_{1}<p_{0}$, which would lead to both an increase in $I C$ and $P C$. In contrast, a variation in $p_{i}$ with $i>2$ influences both the inventory build-up of batch $i$ and the size of the shipments $i$ to $m$. This is due to the fact that the batches 2 to $m$ are produced in the consumption time of the preceding batch, wherefore a decrease (increase) in $p_{i}$ with $i>2$ leads to a lower (higher) batch size and consequently lower (higher) inventory carrying costs. Thus, we can conclude that $p_{1}^{*} \in\left[p_{0} ; p_{\max }\right]$ and $p_{i}^{*} \in\left[p_{\min } ; p_{0}\right]$.

\section{Acknowledgement}

The author is grateful to the anonymous referees for their valuable comments and suggestions on an earlier version of this paper. The author further wishes to thank the editorial team and the reviewers for the fast reviewing process.

\section{Literature}

Bogaschewsky R W, Buscher U D, and Lindner G (2001). Optimizing multi-stage production with constant lot size and varying number of unequal sized batches. Omega 29: 183-191.

Buzacott J A and Ozkarahan I A (1983). One- and Two-Stage Scheduling of Two Products with Distributed Inserted Idle Time: The Benefits of a Controllable Production Rate. Naval Research Logistics Quarterly 30: 675-696.

Eiamkanchanalai S and Banerjee A (1999). Production lot sizing with variable production rate and explicit idle capacity cost. International Journal of Production Economics 59: 251-259.

Elhafsi M and Bai S X (1997). The Common Cycle Economic Lot Scheduling Problem with Backorders: Benefits of Controllable Production Rates. Journal of Global Optimization 10: 283-303. 
Gallego G (1993). Reduced production rates in the economic lot scheduling problem. International Journal of Production Research 31: 1035-1046.

Goldratt E M (1988). Computerized shop floor scheduling. International Journal of Production Research 26: 443-455.

Goyal S K (1977). Determination of Optimum Production Quantity for a Two-Stage Production System. Operations Research Quarterly 28: 865-870.

Goyal S K (1994). A note on 'Effect of production cost on shelf life'. International Journal of Production Research 32: 2243-2245.

Higgins P, Le Roy P, and Tierney L (1996). Manufacturing Planning and Control. London: Chapman \& Hall.

Khouja M (1995). The economic production lot size model under volume flexibility. Computers and Operations Research 22: 513-523.

Khouja M (1999). A note on 'deliberately slowing down output in a family production context'. International Journal of Production Research 37: 4067-4077.

Khouja M and Mehrez A (1994). Economic Production Lot Size Model with Variable Production Rate and Imperfect Quality. Journal of the Operational Research Society 45: 14051417.

Larsen C (1997). Using a variable production rate as a response mechanism in the economic production lot size mode. Journal of the Operational Research Society 48: 97-99.

Larsen C (2005). The economic production lot size model extended to include more than one production rate. International Transactions in Operational Research 12: 339-353.

Lee H L, Padmanabhan V, and Whang S (1997). The Bullwhip Effect in Supply Chains. Sloan Management Review 38: 93-102.

Moon D H and Christy D P (1998). Determination of optimal production rates on a single facility with dependent mold lifespan. International Journal of Production Economics 54: 2940.

Moon I, Gallego G, and Simchi-Levi D (1991). Controllable production rates in a family production context. International Journal of Production Research 29: 2459-2470.

Sarker B R and Babu P S (1993). Effect of production cost on shelf life. International Journal of Production Research 31: 1865-1872.

Schweitzer P J and Seidmann A (1991). Optimizing processing rates for flexible manufacturing systems. Management Science 37: 454-466.

Sharma S (2008). On the flexibility of demand and production rate. European Journal of Operational Research 190: 557-561. 
Silver E A (1990). Deliberately slowing down output in a family production context. International Journal of Production Research 28: 17-27.

Szendrovits A Z (1975). Manufacturing cycle time determination for a multi-stage economic production quantity model. Management Science 22: 298-308.

Szendrovits A Z and Drezner S (1980). Optimizing Multi-Stage Production with Constant Lot Size and Varying Number of Batches. Omega 8: 623-629.

Viswanathan S (1995). A note on 'Effect of production cost on shelf life'. International Journal of Production Research 33: 3485-3486.

Viswanathan S and Goyal S K (1997). Optimal cycle time and production rate in a family production context with shelf life considerations. International Journal of Production Research 35: 1703-1711. 


\section{Table 1}

\begin{tabular}{|l|l|l|l|l|}
\hline$\#$ & $h$ & $c_{T}$ & $\left\{a_{0}, a_{1}, a_{2}\right\}$ & $\left\{p_{\min }, p_{\max }\right\}$ \\
\hline 1 & 5 & 200 & $\{1 / 6000,0.12,24\}$ & $\{320,500\}$ \\
\hline 2 & 10 & 200 & $\{1 / 6000,0.12,24\}$ & $\{320,500\}$ \\
\hline 3 & 15 & 200 & $\{1 / 6000,0.12,24\}$ & $\{320,500\}$ \\
\hline 4 & 5 & 400 & $\{1 / 6000,0.12,24\}$ & $\{320,500\}$ \\
\hline 5 & 5 & 200 & $\{1 / 600,1.2,218.4\}$ & $\{320,500\}$ \\
\hline 6 & 5 & 200 & $\{1 / 60,12,2162.4\}$ & $\{320,500\}$ \\
\hline 7 & 5 & 200 & $\{1 / 6000,0.12,24\}$ & $\{340,500\}$ \\
\hline 8 & 5 & 200 & $\{1 / 6000,0.12,24\}$ & $\{320,360\}$ \\
\hline
\end{tabular}


Table 2

\begin{tabular}{|c|l|c|l|l|}
\hline$\#$ & $\boldsymbol{m}^{*}$ & $\boldsymbol{Q}^{*}$ & $\boldsymbol{p}^{*}$ & \multicolumn{1}{c|}{$\boldsymbol{T C}^{*}$} \\
\hline 1 & 4 & 472.07 & 345.14 & 6885.26 \\
\hline 2 & 6 & 481.66 & 320 & 8687.46 \\
\hline 3 & 6 & 393.28 & 320 & 10040.60 \\
\hline 4 & 3 & 493.58 & 349.92 & 8292.36 \\
\hline 5 & 4 & 465.39 & 358.64 & 6915.44 \\
\hline 6 & 4 & 464.82 & 359.87 & 6918.18 \\
\hline 7 & 4 & 472.07 & 345.14 & 6885.26 \\
\hline 8 & 4 & 472.07 & 345.14 & 6885.26 \\
\hline
\end{tabular}




\section{Table 3}

\begin{tabular}{|c|l|c|c|c|c|}
\hline$\#$ & $\boldsymbol{m}^{*}$ & $\boldsymbol{q 1}^{*}$ & $\boldsymbol{Q}^{*}$ & $\boldsymbol{p}^{*}$ & $\boldsymbol{T} \boldsymbol{C}^{*}$ \\
\hline 1 & 7 & 71.30 & 826.66 & 349.52 & 6410.29 \\
\hline 2 & 7 & 52.24 & 586.10 & 346.34 & 8061.51 \\
\hline 3 & 8 & 42.22 & 539.88 & 339.20 & 9325.47 \\
\hline 4 & 5 & 114.74 & 832.24 & 356.09 & 7809.66 \\
\hline 5 & 6 & 73.15 & 721.59 & 359.22 & 6419.91 \\
\hline 6 & 6 & 72.68 & 721.26 & 359.92 & 6420.84 \\
\hline 7 & 7 & 71.30 & 826.66 & 349.52 & 6410.29 \\
\hline 8 & 7 & 71.30 & 826.66 & 349.52 & 6410.29 \\
\hline
\end{tabular}


Table 4

\begin{tabular}{|c|l|c|l|c|}
\hline$\#$ & $\boldsymbol{m}^{*}$ & $\boldsymbol{Q}^{*}$ & \multicolumn{1}{c|}{$\boldsymbol{p}_{\mathbf{1}} \boldsymbol{*}, \ldots, \boldsymbol{p}_{\boldsymbol{m}} \boldsymbol{*}$} & $\boldsymbol{T} \boldsymbol{C}^{*}$ \\
\hline 1 & 5 & 582.63 & $\{366.51,320,320,336.90,352.99\}$ & 6818.53 \\
\hline 2 & 5 & 414.13 & $\{369.12,320,320,324.63,349.85\}$ & 8591.36 \\
\hline 3 & 5 & 338.92 & $\{371.08,320,320,320,347.36\}$ & 9945.83 \\
\hline 4 & 3 & 503.05 & $\{369.23,324.04,349.71\}$ & 8242.14 \\
\hline 5 & 4 & 466.20 & $\{360.67,356.56,357.95,359.32\}$ & 6911.57 \\
\hline 6 & 4 & 464.90 & $\{360.07,359.66,359.80,359.93\}$ & 6917.80 \\
\hline 7 & 4 & 475.69 & $\{366.64,340,340,352.84\}$ & 6851.92 \\
\hline 8 & 5 & 582.23 & $\{360,320,320,336.92,352.99\}$ & 6819.89 \\
\hline
\end{tabular}




\section{Table 5}

\begin{tabular}{|c|l|c|c|l|c|}
\hline$\#$ & $\boldsymbol{m}^{*}$ & $\boldsymbol{q 1}^{*}$ & $Q^{*}$ & \multicolumn{1}{|c|}{$\boldsymbol{p}_{\mathbf{1}}^{*}, \ldots, \boldsymbol{p}_{\boldsymbol{m}} *$} & \multicolumn{1}{|c|}{$\boldsymbol{T} \boldsymbol{C}^{*}$} \\
\hline 1 & 7 & 73.51 & 830.09 & $\begin{array}{l}\{364.16,352.44,344.86,341.58,342.73,348.26, \\
358.07\}\end{array}$ & 6401.01 \\
\hline 2 & 8 & 52.24 & 663.97 & $\begin{array}{l}\{365.85,349.28,337.64,331.09,329.79,333.80, \\
343.03,357.36\}\end{array}$ & 8041.99 \\
\hline 3 & 9 & 43.43 & 605.92 & $\begin{array}{l}\{367.25,347.49,333.11,324.03,320.17,321.48, \\
328.01,339.87,357.18\}\end{array}$ & 9289.88 \\
\hline 4 & 5 & 116.99 & 833.84 & $\{366.53,355.02,350,351.78,360.29\}$ & 7804.21 \\
\hline 5 & 6 & 73.34 & 721.82 & $\{360.42,359.27,358.64,358.52,358.91,359.81\}$ & 6419.26 \\
\hline 6 & 6 & 72.70 & 721.29 & $\{360.04,359.93,359.86,359.85,359.89,359.98\}$ & 6420.77 \\
\hline 7 & 7 & 73.51 & 830.09 & $\begin{array}{l}\{364.16,352.44,344.86,341.58,342.73,348.26, \\
358.07\}\end{array}$ & 6401.01 \\
\hline 8 & 7 & 73.47 & 829.96 & $\begin{array}{l}\{360,352.49,344.90,341.61,342.74,348.27, \\
358.07\}\end{array}$ & 6401.27 \\
\hline
\end{tabular}




\section{Captions for Figures and Tables}

Figure 1: Inventory-time plots for the case of equal-sized batch shipments

Figure 2: Inventory-time plots for the case of unequal-sized batch shipments

Figure 3: Development of batch sizes for the case of a variable production rate

Figure 4: Shape of function (A-6)

Figure 5: Range of values for $p$ that contains the optimal solution

Table 1: Test problems used for computational experimentation

Table 2: Computational results for the rigid case with equal-sized batch shipments

Table 3: Computational results for the rigid case with unequal-sized batch shipments

Table 4: Computational results for the flexible case with equal-sized batch shipments

Table 5: Computational results for the flexible case with unequal-sized batch shipments 


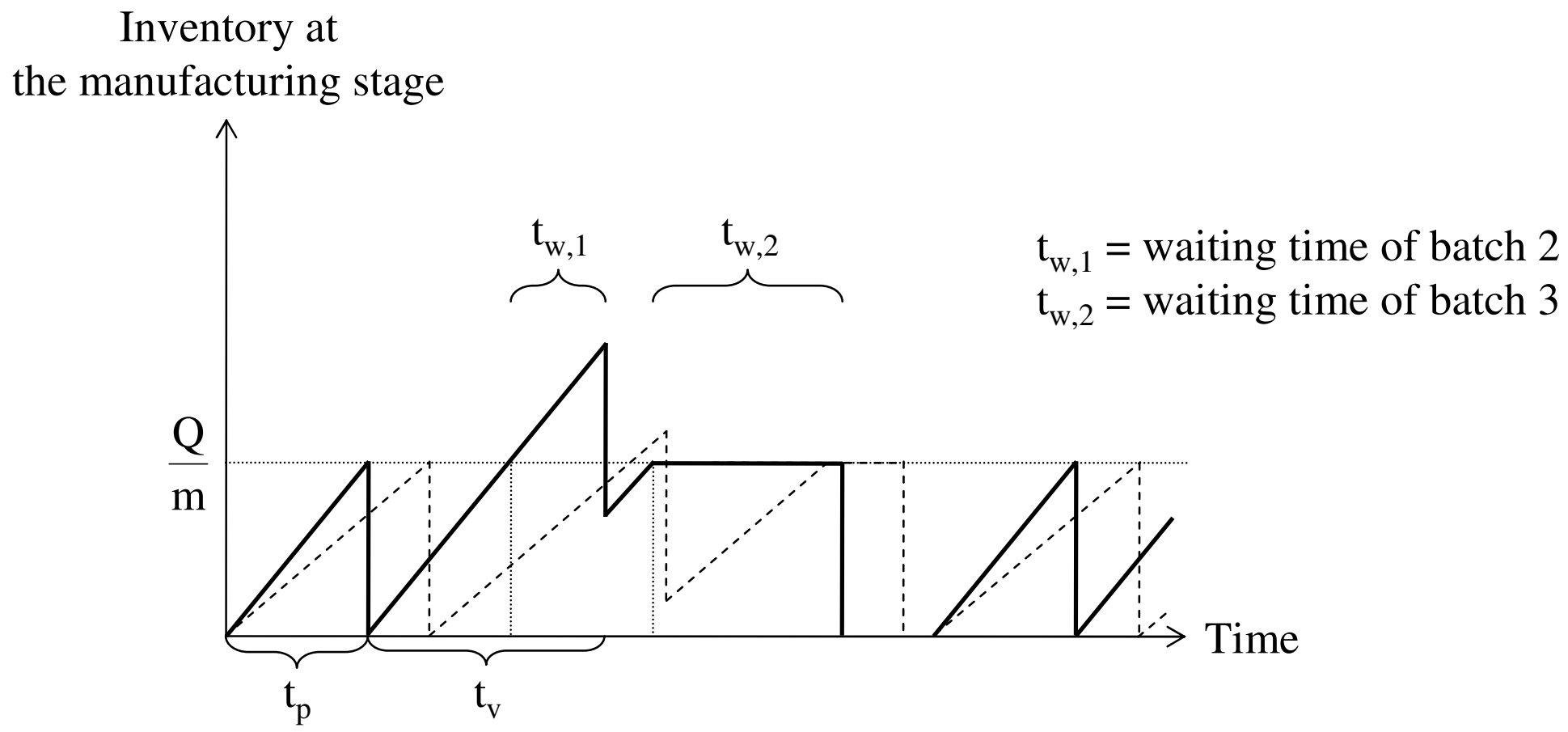

Inventory at

the consuming stage

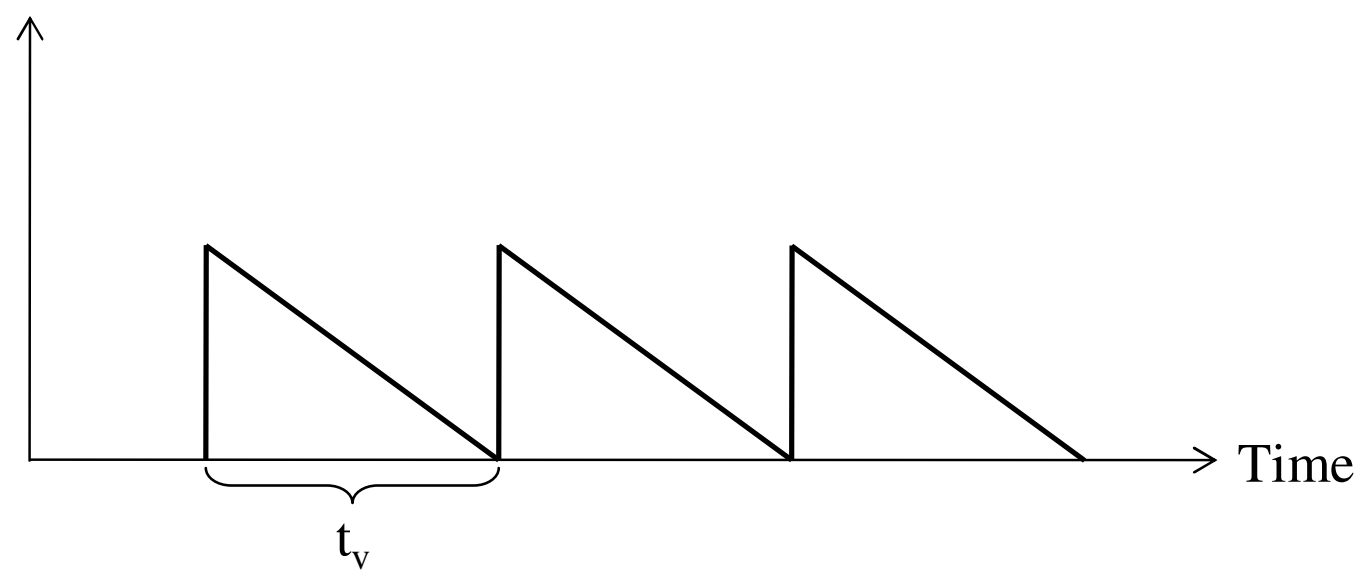


Inventory at

the manufacturing stage

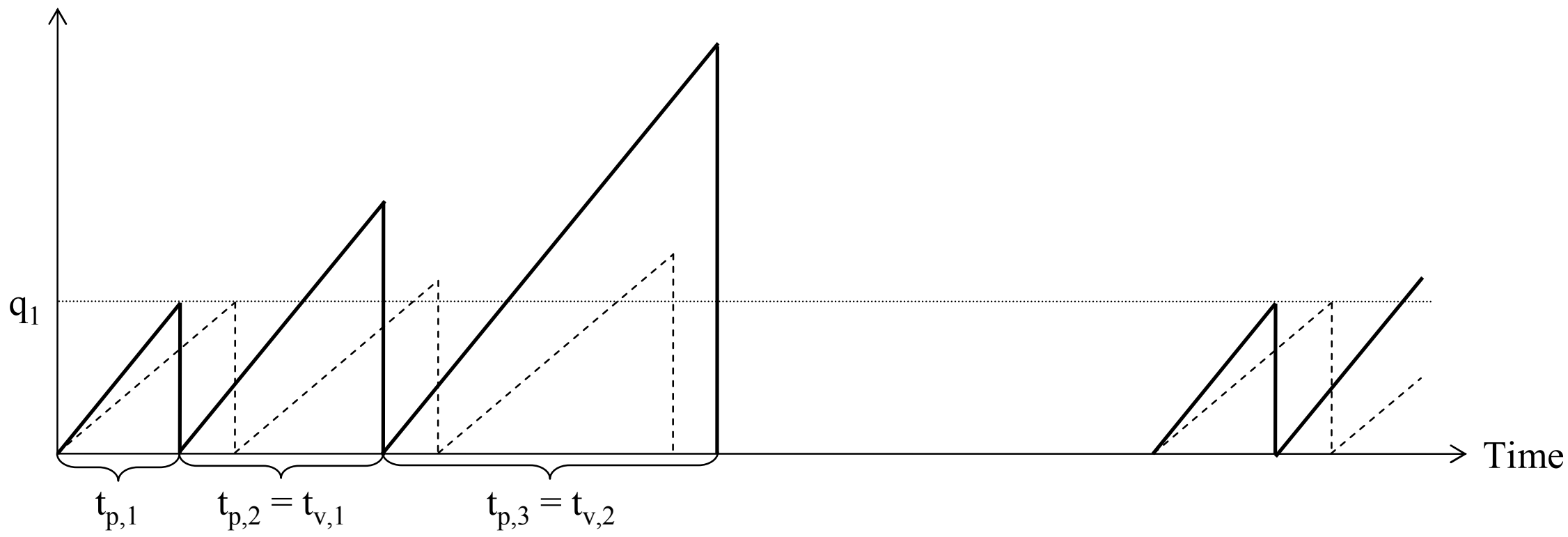

Inventory at

the consuming stage

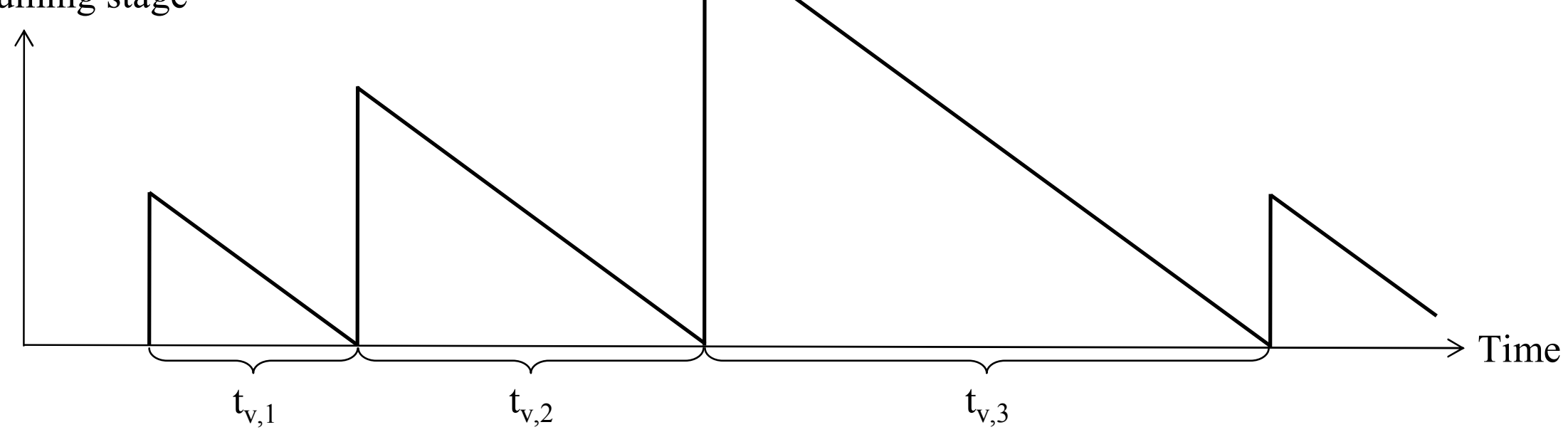

http://mc.manuscriptcentral.com/tprs Email: ijpr@lboro.ac.uk 


\begin{tabular}{|c|c|c|c|c|c|}
\hline size of batch & $\mathbf{\# 1}$ & $\mathbf{\# 2}$ & $\mathbf{\# 3}$ & $\mathbf{\ldots}$ & $\mathbf{\# m}$ \\
\hline & $q_{1}$ & $q_{1} \frac{p_{2}}{d}$ & $q_{1} \frac{p_{2} p_{3}}{d^{2}}$ & $\ldots$ & $q_{1} \prod_{i=2}^{m} \frac{p_{i}}{d}$ \\
\hline
\end{tabular}




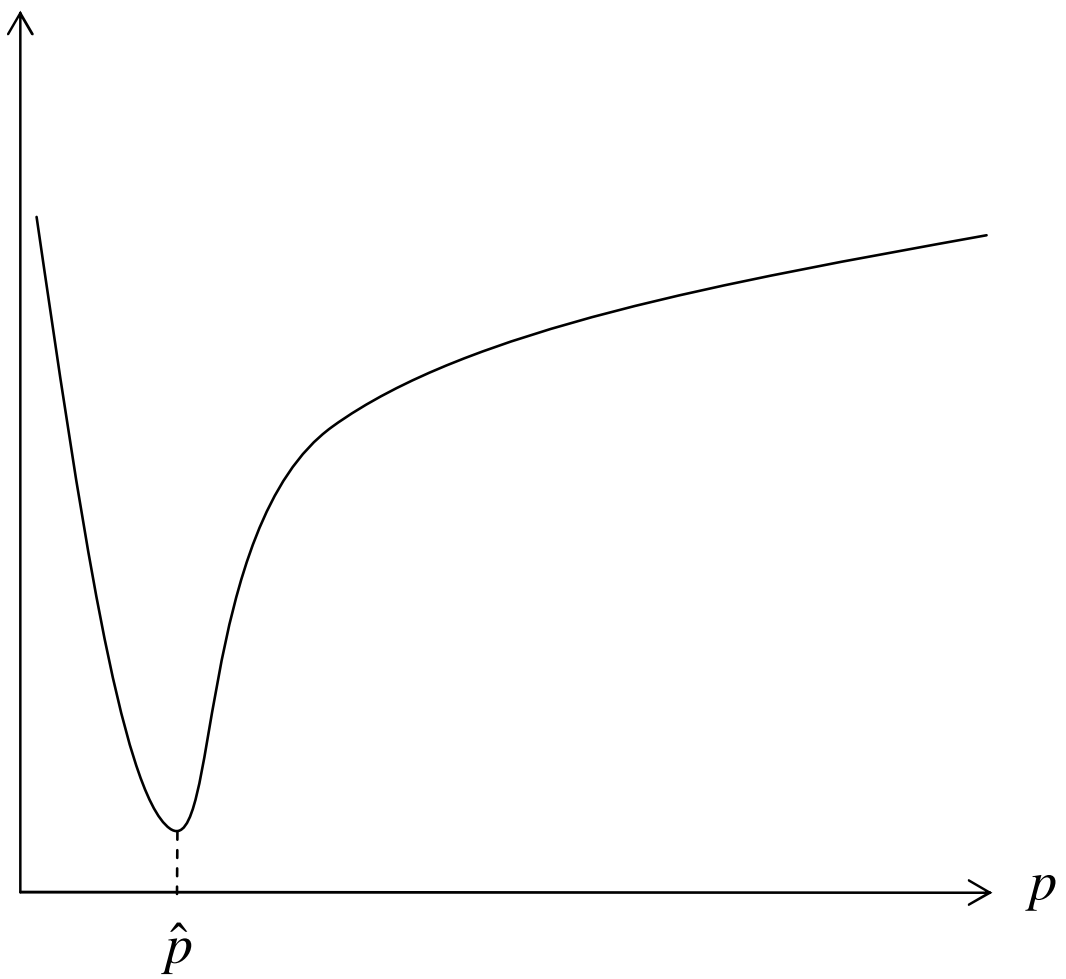



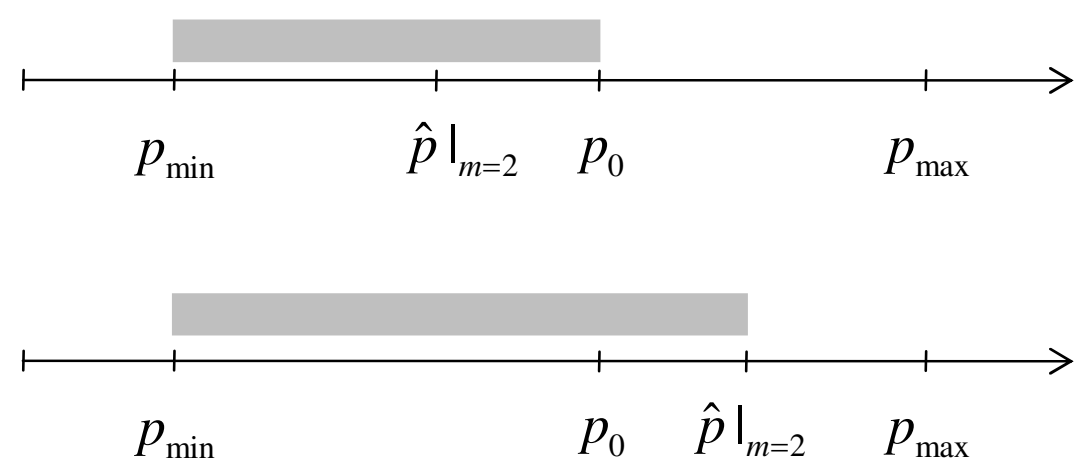

http://mc.manuscriptcentral.com/tprs Email: ijpr@lboro.ac.uk 